\title{
Nitinol Stenting in Post-Traumatic Pseudo-Aneurysm of Internal Carotid Artery
}

\author{
Or Cohen-Inbar $^{1 *}$, Yaaqov Amsalem², Jean F. Soustiel ${ }^{1}$ \\ ${ }^{1}$ Department of Neurosurgery, Rambam Health Care Campus, Technion Israel Institute of Technology, Haifa, Israel \\ ${ }^{2}$ Interventional Neuroradiology Unit, Rambam Health Care Campus, Haifa, Israel \\ Email: *or_coheni@rambam.health.gov.il,"orcoheni@tx.technion.ac.il
}

Received April 9, 2012; revised May 10, 2012; accepted June 6, 2012

\begin{abstract}
Background: A pseudoaneurysm of the internal carotid artery (ICA) at its cervicl part, is a rare and often lethal complication after cervical trauma. The treatment of choice is anticoagulation. Patients with a contraindication for anticoagulation or those who do not improve with standard treatment, warrant either a surgical repair or stent placement. Stent placement posses both short and long term risks such as immediate ischemic events, in-stent stenosis and stent breakage, thus the choice of stent type is critical. Advances in stent technology have made cervical stent placement a viable option. We report our experience with the LEO + (Balt Extrusion, Montmorency, France) nitinol flexible self expanding stent for post-traumatic ICA aneurysms. Methods: 5 patients suffering a post-traumatic cervical ICA pseudo-aneurysms refractory to standard treatment were treated with nitinol braided flexible self expanding stent. This stent has a shape memory and superelasticity virtues making it suitable. Diagnosis was made with CT angiography and confirmed by catheter angiography. All procedures were preformed under local anesthesia. Time between trauma and treatment ranged six days to over five years. Follow-up was performed by CT angiography or conventional angiography. Results: Five patients, having eight aneurysms were treated using 12 stents. In all cases, appropriate stent placement was achieved. No immediate or late complications, as well as no neurological sequele reported at 6 months. Radiological follow-up exams demonstrated complete pseudoaneurysm closure. Conclusion: Stent placement can be a safe and definitive treatment option for patients to conventional medical treatment.
\end{abstract}

Keywords: Interventional Neuroradiology; Angioplasty; Stenting; Nitinol Stent

\section{Introduction}

Traumatic dissection of the internal carotid artery is a well-recognized entity. More frequently diagnosed in recent years due to advances and accebility of tomography in trauma [1,2]. A pseudo-aneurysm of the cervical part of the internal carotid artery (ICA) is a rare and often lethal complication of blunt or penetrating cervical trauma $[3,4]$. Left untreated, these lesions are associated with adverse outcomes, namely hemorrhage, enlargement, occlusion and thromboembolic events [1-4]. Thus, prompt diagnosis and treatment is essential. Historically speaking, most pseudoaneurysms have been treated surgically in accessible lesions. Non-accessible lesions were treated with anticoagulation and antiplatelet therapy, rarely bringing resolution [1,3,5]. $40 \%$ of traumatic dissections and the majority of pseudo-aneurysms do not heal with anticoagulant therapy, thus presenting with a long-term risk of embolic or flow-related complications [1,2,5]. Those patients in which there is a contraindication for anticoagulation or have not improved with standard treatment

\footnotetext{
"Corresponding author.
}

warrant either a surgical repair or stent placement. Carotid stenting has been used for the treatment of carotid athero-sclerotic disease $[1,5,6]$. The ability to place these stents, using endovascular techniques, has permitted extrapolation of their use to other patient populations. Once proved as a feasible alteranative, stenting of posttraumatic pseudoaneurysms is appealing, since the immediate revascularization achieved may obviate the need of anticoagulation [2-4]. Still, carotid stenting posees both short and long term risks. Immediate ischemic events, instent stenosis as well as stent breakage to name a few. The choice of stent type is thus critical. Self-expanding stents are preferred in the carotid artery because of the potential for permanent collapse of the stent from extrinsic pressure $[1,5,6]$.

Nitinol (Nickel-Titanium) alloys exhibit a combination of properties making these alloys particularly suited for selfexpanding stents [8]. Nitinol stents are self-expanding without the need for post-deployment heating. Nitinol stents are superelastic, hence crush recoverable $[5,6,8]$. The nitinol stents exert a gentle chronic outward force and are 
generally more physiologically compatible than balloonexpandable stents [8]. Braided stents offer several additional advantages to the conventional self expanding stents, such as the better wall apposition, smaller pores resulting in a flow diverting effect and better radio-opaque markers $[5,6]$. We report our experience with Nitinol flexible self expanding stent (LEO +, Balt Extrusion, Montmorency, France) for the treatment of post-traumatic ICA pseudoaneurysms.

\section{Material and Methods}

All head trauma patients underwent a routine admission noncontrast brain computed tomography, cervical spine and carotid imaging, as well as any other imaging required by clinical suspicion. Patients with neurological signs or radiological evidence of injury, with penetrating injury or otherwise unstable, were hospitalized. 5 patients suffering a post-traumatic cervical ICA pseudo-aneurysms refractory to standard treatment were treated with nitinol flexible self expanding stent. Diagnosis was made with CT angiography and confirmed by catheter cervical angiography. All procedures were preformed under local anesthesia. Time interval from injury to stent treatment varied from six days to over five years after the cervical trauma, depending on the clinical presentation and general medical condition. Board-certified neurosurgeons and intereventional neuro-radiologists logged the clinical considerations and procedure data. All patients were treated post-procedure with clopidogrel (75 mg daily) and aspirin (100 mg daily). Follow-up was performed by CT angiography or conventional angiography. Outcome data was collected at several time points after the intervention. The out-patient clinic charts and medical records from further hospitalizations were used for long-term follow-up.

\section{Results}

Five patients revealed to suffer with eight aneurysms were treated using twelve nitinol stents. See Table 1 for patient's demographic data and Table 2 for patient's outcome data. In all cases described, appropriate stent placement was achieved (Figures 1-4). No immediate or late complications noted, no neurological sequele reported at 12 months. Radiological follow-up exams demonstrated complete aneurysm closure (Figures 1-4).

\section{Discussion}

A pseudo-aneurysm of the cervical part of the internal carotid artery (ICA) is a rare and often lethal complication of blunt or penetrating cervical trauma. Left untreated, these lesions are associated with adverse outcomes, namely hemorrhage, enlargement, occlusion and thromboembolic events. Thus, prompt diagnosis and
Table 1. Patients demographic data.

\begin{tabular}{ccccc}
\hline Patient no. & Age (years) & Sex & Type of injury & No. of stents placed \\
\hline 1 & 40 & M & MVA $^{*}$ & $2\left(2{ }^{* *} \mathrm{PA}\right)$ \\
2 & 21 & $\mathrm{~F}$ & MVA & $1(1 \mathrm{PA})$ \\
3 & 57 & $\mathrm{M}$ & War & $6(3 \mathrm{PA})$ \\
4 & 18 & $\mathrm{M}$ & MVA & $1(1 \mathrm{PA})$ \\
5 & 86 & $\mathrm{~F}$ & Head trauma & $2(1 \mathrm{PA})$ \\
\hline
\end{tabular}

${ }^{*}$ MVA = Motor vehicle accident; ${ }^{* *} \mathrm{PA}=$ Pseudoaneurysm.

Table 2. Treatment outcome data.

\begin{tabular}{|c|c|c|c|c|}
\hline \multirow{2}{*}{$\begin{array}{l}\text { Patient } \\
\text { no. }\end{array}$} & \multirow{2}{*}{$\begin{array}{l}\text { Mean follow-up } \\
\text { (months) }\end{array}$} & \multicolumn{2}{|c|}{ Complications } & \multirow{2}{*}{$\begin{array}{c}\text { Procedure } \\
\text { outcome }\end{array}$} \\
\hline & & Short term & Long term & \\
\hline 1 & 15 & None & None & ${ }^{*} \mathrm{Cc}$ \\
\hline 2 & $\mathrm{n} / \mathrm{a}$ & None & $\mathrm{n} / \mathrm{a}$ & Cc \\
\hline 3 & 7 & None & None & Cc \\
\hline 4 & 5 & None & None & Cc \\
\hline 5 & 4 & None & None & Cc \\
\hline
\end{tabular}

${ }^{*} \mathrm{Cc}=$ Complete closure.

treatment is essential [1-4]. Historically speaking, most pseudoaneurysms have been treated surgically in accessible lesions. Nonaccessible lesions were treated with anticoagulation and antiplatelet therapy, rarely bringing resolution [1-4].

In recent years endovascular techniques have become more appealing as multiple studies have provided encouraging results $[5,6,8]$. Coldwell et al. [1] describe 14 patients with traumatic ICA pseudoaneurysms treated with self-expanding bare metal stents. Two patients had persistent pseudoaneurysms at 2-month follow-up, which eventually resolved 2 months, the rest had complete resolution on follow-up. Bush et al. [2] reported the treatment of ICA pseudoaneurysms by placing bare stents and adding coil embolization of the excluded pseudoaneurysm in 5 patients, with satisfying results at follow-up (mean 11.7 months). 1 of the 4 patients developed a 60\% focal stenosis. However, even with secondary coiling through a bare stent, Layton et al. [4] showed that an ICA pseudoaneurysm can continue to opacify and enlarge. Patel et al. [6] have stated that there is always a potential for pseudoaneurysm recanalization when using bare stents, even with secondary coiling of the pseudoaneurysm. Self-expanding stents are preferred in the carotid artery because of the potential for permanent collapse of the stent from extrinsic pressure $[5,6]$.

Dieter Stoeckel et al. [8] presented the nitinol stents in 2003, stating that Charles's Dotter experiments with Nitinol wire coils as intra-arterial scaffolds in the early nineteen eighties, distinguished Nitinol for its unusual shape memory effect $[5,6,8]$. Nitinol (Nickel-Titanium) alloys was shown to exhibit properties which make it particularly suited for making self expanding stents. Today, twenty five years after Dotter's experiments, Nitinol stents 


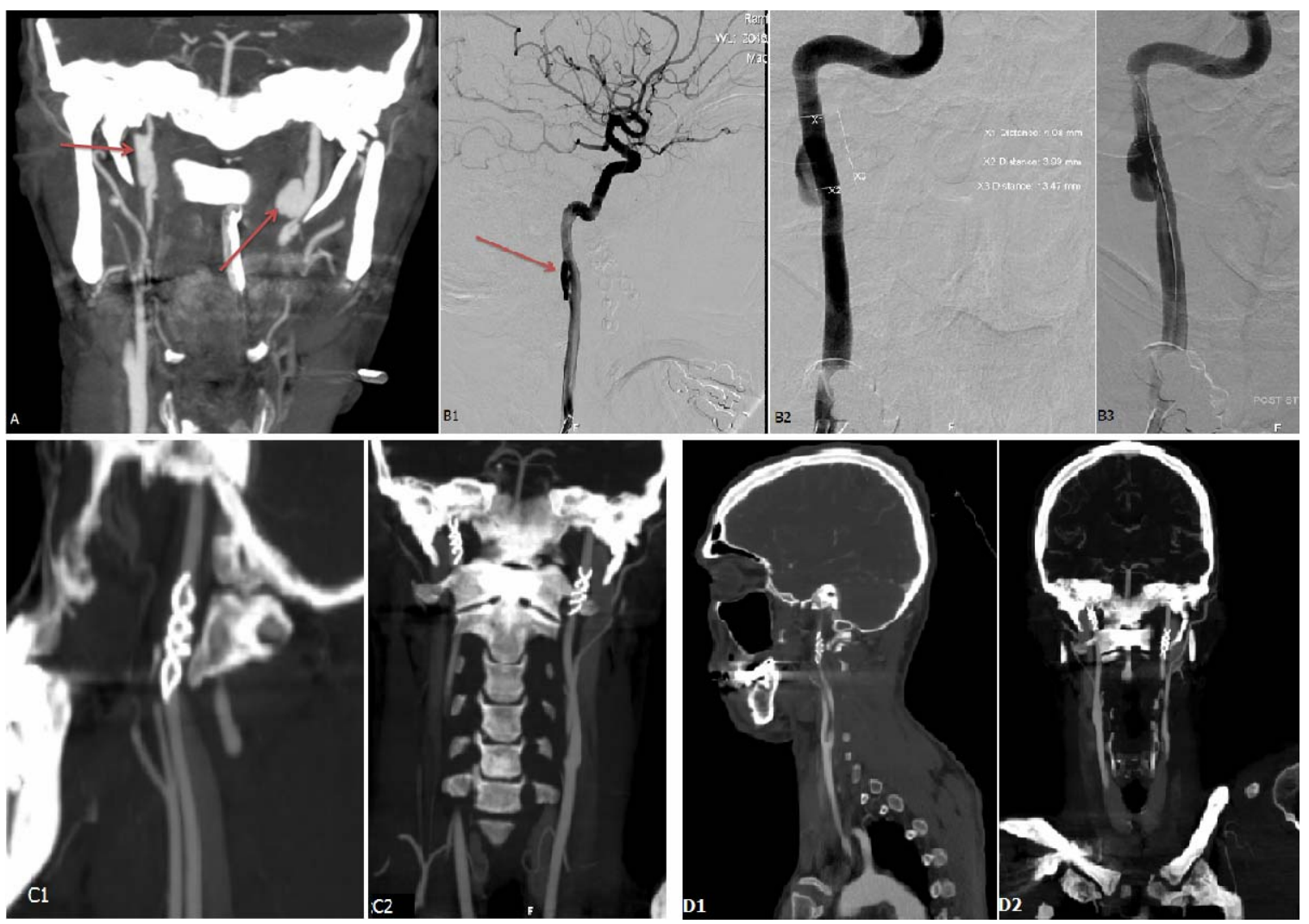

Figure 1. Images of patient \#1 in table \#1. (A) Coronal mip, showing bilateral cervical ICA pseudoaneurysm, pre-stenting scan. Angiography images (B1) showing injection into Right ICA demonstrating the pseudo-aneurysm. Planning (B2) and placing (B3) of the stent. Follow-up tomography post stenting, immediate coronal and sagital (C), and 5 month follow-up (D).

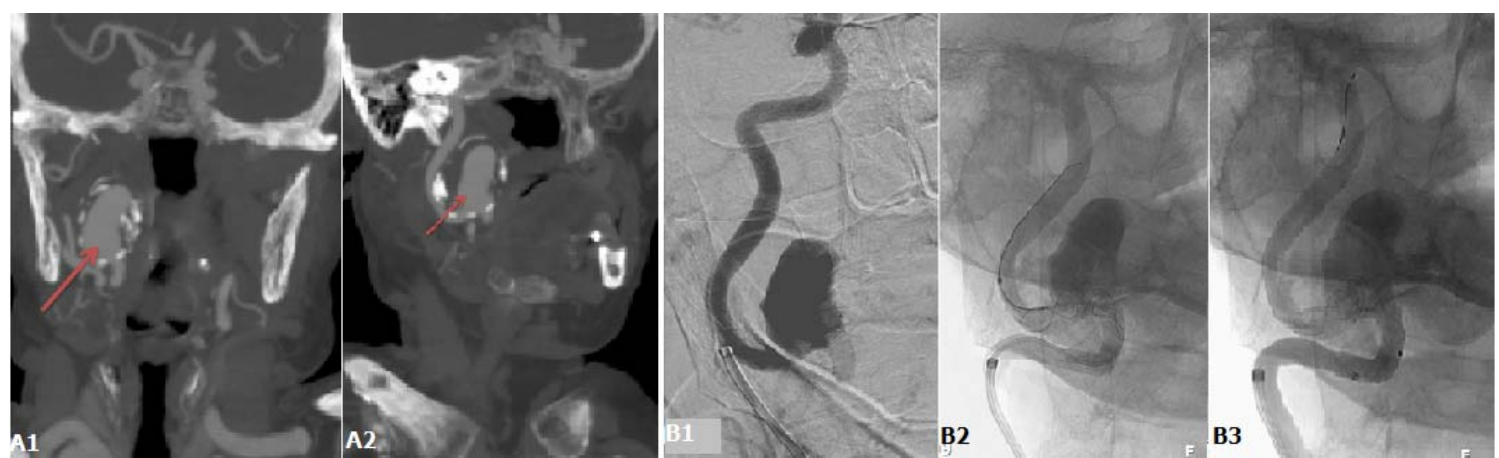

Figure 2. Images of patient \#5 in table \#1. (A) CT angio, showing a large long standing pseudoaneurysm in a tortuous Right ICA; (B) Angiography, Stent placement $\times 2$.

are self-expanding without the need for post-deployment heating. Nitinol stents are superelastic, hence crush recoverable. These stents are manufactured to a size slightly larger than the target vessel size and delivered constrained in a delivery system. After deployment they position themselves against the vessel wall with a low, chronic outward force. The stents resist outside forces with a significantly higher radial resistive force. Despite the high nickel content of Nitinol, its corrosion resistance and biocompatibility is equal to that of other implant materials. Conventional stent materials, like stainless steel or cobalt based alloys, have a different elastic profile than biological materials. The elastic deformation of these metals is limited to approximately $1 \%$ strain, and elongation typically increases and decreeases proportionally with the applied force. Natural materials on the other hand, like hair, tendon and bone can be elastically deformed, in some cases up to $10 \%$ strain in a non-linear way [7]. As shown in Figure 4(a), the loading/unloading cycle is characterized by a pronounced hysteresis. Figure 4(b) shows a characteristic stress/strain curve for a Nitinol alloy wire at body temperature. 

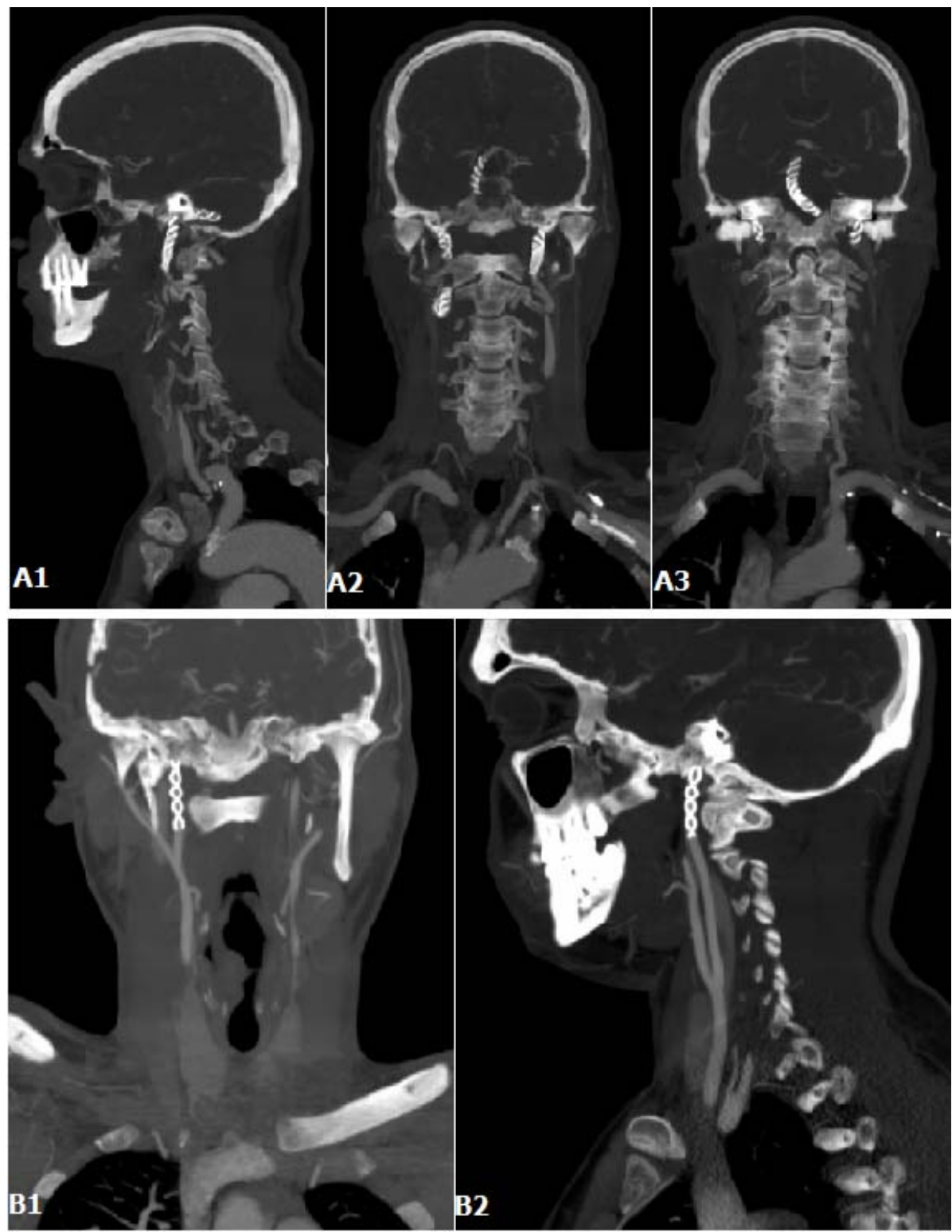

Figure 3. (A1)-(A3) Coronal ans sagittal MIP of patient \#2 in table \#1, 5 months follow-up post stenting; (B1) and (B2) Coronal and sagittal MIP of patient \#3 in table \#1, 5 months follow-up post stenting.

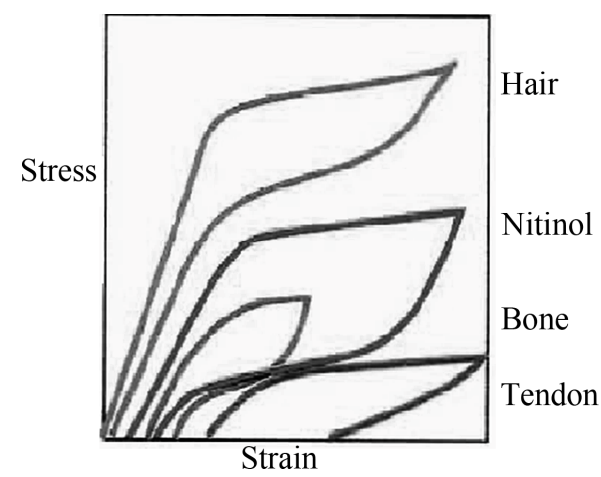

(a)

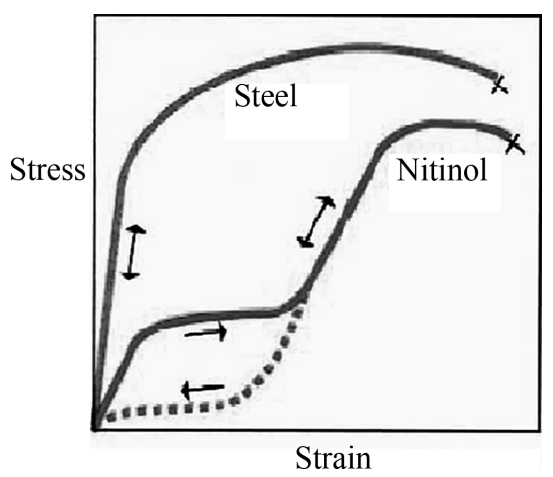

(b)

Figure 4. Biomechanical compatibility of Nitinol. (a) Deformation characteristics of Nitinol and living tissues; (b) Schematic stress-strain diagram for Nitinol and stainless steel. 
The mechanical and biophysical features of the nitinol stents have withstand the test of reality, proving to be a convient and easy to handle during the procedure. An important feature of the nitinol self expanding stents lies in it being a braided stent. This fact allows for several advantages over other self-expanding stents, pertinent to our goals, first of which is a better wall apposition of the stent to the arterial wall. Early reports, regarding older generation LEO stents described to show flattening of the stent midsection and inward crimping of the proximal and distal ends, were shown to not exist in the newer generation LEO plus nitinol stents [6]. The LEO plus stent showed symmetric deployment at all tested degrees of curvature, without flattening or kinking. The stent retained its round cylindrical shape at all curvatures without inward crimping of its proximal and distal ends. This distinguishes these braided stents from the other (open-cell or closed-cell) LAZER-cut self expanding stents $[5,6,8]$. A second feature of the braided nitinol stent is in its smaller pore size as compared to the other self-expanding stents, resulting in a more dominant flowdiversion effect. A third advantage of the LEO plus stent stems from the better radio-opaque markers positions, allowing to better visualize stent deployment throughout its length, and thus correction of a suboptimally deployd stent.

The short five patients series described is characterized by a single angiographic therapeutic procedure with a good outcome, both clinically and radiologically, in a very diverse cohort. Immediate clinical results, measured in patient's neurological disability and neurological sequale, together with different aspects of quality of life and radiologically were satisfying. Immediate tomographic and angiographic, as were the long term follow-up images, patient condition and patient satisfaction are satisfying.

\section{Conclusion}

The LEO + (Balt Extrusion, Montmorency, France) nitinol braided self expanding stent seems to be a safe and definitive treatment option for post-traumatic cervical ICA pseudoaneurysms. It should be considered in patients unresponsive to standard treatment, or those developing complications or presenting contraindications for chronic anticoagulation. These encouraging results have spurred further indications for nitinol stenting. Traumatic dissec- tions of the vertebral arteries, stenting of the carotid artery in established acute thrombosis and stroke or transient ischemic attack are currently being treated with these stents at our institute. Further study and evaluation is required, and a proper report of our experience is forthcoming.

\section{REFERENCES}

[1] D. M. Coldwell, Z. Novak, R. K. Ryu, et al., "Treatment of Posttraumatic Internal Carotid Arterial Pseudoaneurysms with Endovascular Stents," Journal of TraumaInjury Infection \& Critical Care, Vol. 48, No. 3, 2000, pp. 470-472. doi:10.1097/00005373-200003000-00016

[2] J. V. Patel, M. M. Rossbach, T. J. Cleveland, P. A. Gaines and J. D. Beard, "Endovascular Stent-Graft Repair of Traumatic Carotid Artery Pseudoaneurysm,” Clinical Radiology, Vol. 57, No. 4, 2002, pp. 308-311. doi:10.1053/crad.2001.0808

[3] C. T. Dotter, R. W. Buschmann, M. K. McKinney and J. Rosch, "Transluminal Expandable Nitinol Coil Stent Grafting: Preliminary Report,” Radiology, Vol. 147, No. 1, 1983, pp. 259-260.

[4] K. F. Layton, Y. W. Kim and J. H. Hise, "Use of Covered Stent Grafts in the Extracranial Carotid Artery: Report of Three Patients with Follow-Up between 8 and 42 Months,” American Journal of Neuroradiology, Vol. 25, No. 10, 2004, pp. 1760-1763.

[5] R. L. Bush, P. H. Lin, T. F. Dodson, J. E. Dion and A. B. Lumsden, "Endoluminal Stent Placement and Coil Embolization for the Management of Carotid Artery Pseudoaneurysms," Journal of Endovascular Therapy, Vol. 8, No. 1, 2001, pp. 53-61. doi:10.1583/1545-1550(2001)008<0053:ESPACE $>2.0$.C $\underline{\mathrm{O} ; 2}$

[6] M. Alvarado, N. Ebrahimi and G. Benndorf, "Study of Conformability of the New LEO Plus Stent to a Curved Vascular Model Using Flat-Panel Detector Computed Tomography (DynaCT),” Neurosurgery, Vol. 64, No. 3, 2009, pp. 130-134.

[7] S. A. Shabalovskaya, "On the Nature of the Biocompatibility and on Medical Applications of NiTi Shape Memory and Superelastic Alloys,” Bio-Medical Materials and Engineering, Vol. 6, No. 4, 1996, pp. 267-289.

[8] D. Stoeckel, A. Pelton and T. Duerig, "Self-Expanding Nitinol Stents: Material and Design Considerations,” European Radiology, Vol. 14, No. 2, 2004, pp. 292-301. doi:10.1007/s00330-003-2022-5 